\title{
Etude des particules discursives dans des situations de parole particulière : les scénarios et leurs oralisations par les acteurs
}

\author{
Sandra Teston-Bonnard \\ UMR ICAR \\ Sandra.Teston-Bonnard@univ-lyon2.fr
}

\section{Introduction}

\subsection{Elaboration d'une situation de parole particulière : les dialogues de cinéma}

Depuis des décennies, du dialogue théâtral au langage du cinéma, les auteurs cherchent à « (re)trouver » une parole qui puisse donner à leurs œuvres la couleur du réalisme.

Des dramaturges s'intéressent depuis longtemps à une «parole en éclats » dont la distribution par répliques obéit moins à la nécessité de construire un discours qu'à celle de saisir le mouvement de la parole, ses flux et ses reflux, ses hésitations, ses ratages et ses obsessions (...) (Ryngaert, 1993)

La vraie question demeure celle de l'adéquation de la langue au réel (Ryngaert, id.)

Mais est-ce justement « la vraie question »? D’une part, les dialogues de cinéma peuvent être très « écrits ${ }^{1}$ et, il est alors difficile de retrouver les aspects décrits par les linguistes spécialistes de l'oral qui travaillent à partir de données attestées ${ }^{2}$. D'autre part, quand les dialogues de cinéma paraissent se rapprocher fortement d'un des aspects de la langue orale que tout locuteur pourrait intuitivement identifier, il faut se demander dans quelle mesure ces dialogues cinématographiques-là sont réellement proches de certaines des situations de parole répertoriées et étudiées à partir de nos banques de données.

\section{Chion (2008:9) remarque :}

En français, par rapport à d'autres langues comme l'anglais, le style neutre ${ }^{3}$ est sinon existant, du moins faible et rare. En d'autres termes, nous sommes obligés la plupart du temps de choisir entre une formulation qui peut être trop précieuse, ou guindée, ou savante, et une autre qui peut être trop familière ou désinvolte, voire vulgaire.

\section{$1.2 \quad$ Méthodologie}

Pour analyser les aspects de la langue utilisée dans certains films de cinéma, la méthodologie choisie a permis, d'abord, de confronter le travail du scénariste à celui de l'acteur - le texte proposé par l'auteur et l'oralisation transcrite de l'acteur -, et, au cours de l'étape suivante, de comparer les résultats issus de l'analyse des corpus cinématographiques étudiés ici avec ceux des données attestées des grands corpus $\mathrm{d}^{\prime}{ }^{\prime}{ }^{4}{ }^{4}$. Cette méthodologie nous paraît suffisamment inédite pour proposer cet apport aux travaux de recherche réalisés jusque là sur le langage cinématographique, ou dans le cadre plus général de l'étude du français parlé.

Le corpus de cinéma exploité dans cette contribution est constitué de deux films, de leurs versions écrites et de leurs versions orales. Il s'agit de Un Air de Famille et Le Goût des Autres, écrits et interprétés par A. Jaoui et J.-P. Bacri (réalisation de C. Klapisch pour le premier, d'A. Jaoui pour le second) dont nous avons étudié les scénarios ou, plus exactement, les versions écrites ${ }^{5}$ et les versions oralisées. Les dialogues interprétés par les acteurs ont été transcrits selon les conventions du GARS ${ }^{6}$. Si nous avons centré cette étude sur le travail de A. Jaoui et de J.-P. Bacri, c'est parce qu'il nous est apparu, 
intuitivement, que leurs dialogues pouvaient être rapprochés assez facilement des situations de parole qu'ils sont censés montrer. Il était donc intéressant d'observer quels aspects, quels indices peuvent produire cet effet de «naturel » qu'en tant que spectateurs nous ressentons. Il est en effet impossible pour un auteur de reproduire des interactions comme elles se construisent réellement dans la vie : le film perdrait vite de son intérêt $t^{7}$ ! Il suffit d'écouter les corpus oraux de conversations que nous enregistrons dans nos laboratoires pour le comprendre.

Le choix de ces films est aussi lié à la méthode de travail de leurs auteurs. Après avoir écrit les dialogues, ceux-ci les « disent»; ils se les réapproprient oralement, puis ils réécrivent les textes d'après ce travail d'oralisation (les auteurs ont expliqué très fréquemment leurs méthodes de travail dans des entretiens médiatisés).

\subsection{L'objet d'étude épistémologique traité sous l'angle de la «syntaxe interactionnelle »}

Nous avons sélectionné et traité un fait particulier de l'oral : les petits mots du discours, tels que Ducrot les nommait, et auxquels nous attribuons l'appellation de Particules Discursives (PDi).

A partir d'une analyse comparée des PDi relevées dans les deux scénarios de cinéma et dans des corpus de données attestées de conversation, il est alors possible de répondre aux objectifs méthodologiques évoqués supra : d'abord, en comparant les PDi observées dans le texte du scénario et dans l'oralisation qu'en ont fait les acteurs; ensuite, en confrontant ces résultats aux relevés qui avaient été effectués sur corpus oraux évoqués supra (voir note 4) pour des travaux sur le statut des particules discursives (TestonBonnard, 2006, 2007).

Nous nous sommes penchée en premier lieu sur les définition et le statut grammatical des unités qui nous intéressent ici : si les entrées des dictionnaires semblent souvent incomplètes, voire absentes, qu'en est-il de la place des PDi dans les grammaires? La plupart des auteurs considèrent qu'il n'est pas pertinent de les étudier sous l'angle syntaxique :

Leur fonction syntaxique est nulle (...) (D. Vincent, id., p. 60)

Les ponctuants ont des origines diverses mais doivent tous avoir subi une perte de valeur syntaxique, référentielle et expressive (D. Vincent, id., p. 63)

Les analyses contemporaines qui les décrivent, proviennent toujours du domaine de l'énonciation, de la pragmatique interactionnelle, du discours et de la prosodie.

[...] nous abordons la description de ben dans une perspective interactionniste, qui s'organise autour de la notion centrale de co-construction. En effet, si ce petit mot a été choisi a priori, nous ne l'abordons pas pour contribuer à l'étude d'une partie du discours, interjection, voire adverbe (cf. Petit Robert), mais en tant que ressource que les locuteurs utilisent largement (Bruxelles \& Traverso, $2001: 39$ ).

Comme l'ont remarqué avec pertinence Bruxelles et Traverso (2001), les classements proposés par la tradition (quand un classement est proposé !) concernent la catégorie grammaticale de l'unité considérée, quitte pour les auteurs de dictionnaires ou de grammaires à utiliser une démarche $a d$ hoc, et aucune fonction ne lui est attribuée. Même les approches de Blanche-Benveniste et du GARS, qui ont évoqué ces éléments dans une perspective « intono-syntaxique » sous l'appellation de phatiques (1990:146,) n'en ont jamais fait une étude systématique. Quelle que soit l'emploi de la forme, les grammaires traditionnelles conservent les dénominations suivantes en termes de catégorie grammaticale :

adverbe pour enfin, alors, là (dans la majorité des ouvrages de grammaire de référence), préposition ou de présentatif-introducteur pour voilà (Goosse dans Grevisse), interjection pour bon (Riegel, p. 464), si, bien sûr, la catégorie « interjections » est présentée dans ces ouvrages ; on a déjà dit que, souvent, l'emploi de ces formes à ce niveau- là n'est en fait jamais évoqué, par exemple le « pronom » quoi dans son emploi discursif, incises ou d'incidentes pour tu vois, tu sais. 
Les manuels de grammaire n'évoquent généralement pas $b^{8}{ }^{8}$ (la forme ben n'a pas d'autre emploi que la fonction discursive : on peut en effet montrer que bien n'est pas substituable à $\left.b e n^{9}\right)$, ni les locutions de type je veux dire. Nous savons que dans les corpus de français parlé les emplois «discursifs" sont beaucoup plus fréquents que les emplois grammaticaux pour les formes bon, quoi, enfin (Chanet, 2004), et ce ne sont pourtant pas ceux-là qui sont les plus étudiés. Pour les unités qui nous intéressent, nous trouvons la même proportion d'emplois grammaticaux traditionnels et d'emplois discursifs dans les corpus de cinéma exploités ici que dans les autres corpus de données authentiques ; nous proposons donc de réaliser le travail que celui que nous avons déjà effectué à partir des autres corpus, en examinant comment les PDi produites dans le langage de cinéma se comportent syntaxiquement, et si les mêmes organisations sont alors repérables.

Il n'est pas envisageable de traiter ces éléments en termes micro-syntaxiques puisqu'ils ne sont pas définissables par des relations grammaticales clairement identifiées : ils ne sont pas régis par le verbe principal, ni par un autre constituant lui-même régi par le verbe, ils ne sont ni compléments, ni spécifieurs, ni ajouts. Pour leur description, nous utilisons les unités de la macro-syntaxe aixoise (Blanche-Benvensite, 1990) liées aux concepts interactionnels d'engagement, de langage en situation, de conduites ordonnées obéissant à des règles de procédure : co-construction, énoncés collaboratifs, rôle des PDi dans la structure et la construction de l'interaction, hiérarchisation des informations, gestion des tours, planification, projection (Mondada, 1995, 2001, 2004, notamment ; Traverso, 1999, 2002, 2005).

\section{Examen des différences relevées entre des corpus de langage de cinéma et des autres corpus de données authentiques}

\subsection{Traitement et usages des PDi par les auteurs et par les acteurs}

Comme les PDi, les hésitations, les « bégaiements », les répétitions, les « inachèvements », les recherches de dénominations, les chevauchements, etc., toutes les turbulences, les «scories» de l'oral, sont les « traces de composition (du langage)

[...] passionnant sujet d'observations; on y voit la production du langage en train de se faire ; les processus que nous utilisons ne sont pas propres à un individu ; ils sont suffisamment généraux pour que l'on puisse y trouver des règles de production qui concernent tous les locuteurs. (Blanche-Benveniste, 1990).

Dans un objectif qui vise à enrichir la description de la langue utilisée dans nos corpus, nous avons donc cherché la présence des PDi dans le scénario, et examiné ce qu'en font les acteurs, afin de pouvoir dégager des règles de production sur le plan syntaxique et interactionnel.

\subsubsection{Prédictibilité des PDi}

Nous avons étudié les particules discursives les plus fréquentes dans les corpus étudiés, bon, ben, voilà, enfin, alors, quoi, là, je veux dire, tu sais, tu vois, comme des éléments participant à la production d'une langue qui se construit pas à pas dans l'interaction.

[...] ceux dont le discours est reçu comme convaincant, clair et agréable à suivre, ne parlent justement pas -ou du moins pas forcément- comme des livres. A contrario, il peut être éprouvant pour un auditeur de suivre un conférencier qui lit un texte sans jamais ni s'interrompre ni se répéter. (Béguelin, 2000)

Dans un premier temps, nous avons observé les places et la distribution de ces PDi dans nos corpus de cinéma. Il est remarquable de constater que les auteurs ont inséré des particules discursives dans les dialogues écrits, pour que la situation du film, -conversations familières en famille, entre amis, entre collègues-, soit représentée par une parole qui sera reçue par le spectateur comme adaptée au contexte. Nous avons étudié les places prévues pour ces PDi «écrites», afin de confronter ces résultats à ceux dégagés par l'étude des autres corpus de données authentiques sur l'organisation de ces éléments. Les acteurs ont donc à leur disposition un texte tout fait, le scénario, les dialogues qu'ils doivent apprendre. Or, ils produisent des particules discursives en disant leur texte. Il était particulièrement intéressant 
d'observer si les acteurs reproduisaient les PDi écrites, et de quelle manière ils le faisaient. Les reprenaient-ils exactement, ou bien s'en débarrassaient-ils pour en produire d'autres ailleurs ? Pouvait-on rapprocher ces productions de l'acteur (organisées, là aussi) des fréquences et des régularités distributionnelles déjà repérées dans l'oral de conversation par les linguistes ? L'étude porte aussi sur l'existence éventuelle de variantes des formes PDi utilisées : qu'indique l'acteur quand il choisit une autre PDi que celle qui était prévue? Le changement indique-t-il des paradigmes de variantes ou des variétés dont l'emploi serait aléatoire?

Dans ces interactions spécifiques, il sera également intéressant de s'interroger sur les phénomènes qui ne se produisent pas, alors qu'on les rencontre dans d'autres corpus.

Nous ne donnons ici les résultats les plus significatifs, seulement pour l'un des films étudiés (faute de pouvoir, pour les quatre formes PDi les plus fréquentes $(92 \%$ des occurrences totales de PDi) dans le corpus :

Quelques particules discursives : fréquences dans Un Air de Famille

\begin{tabular}{lcccc}
\hline & BEN & BON & HEIN & ALORS \\
\hline Prévues (scénario) & 15 & 27 & 15 & 18 \\
\hline Reprises idem (acteur) & $\mathbf{1 2}$ & $\mathbf{2 4}$ & $\mathbf{1 5}$ & $\mathbf{1 6}$ \\
\hline Introduites ailleurs (acteur) & 58 & 17 & 49 & 6 \\
\hline PDi produites en totalité (acteur) & 73 & 44 & 64 & 24 \\
\hline
\end{tabular}

Tableau 1

Bien qu'ils n'aient pas de place fixe dans l'énoncé (...) (D. Vincent, p. 61)

[...] on ne peut pas présupposer des endroits où on aurait pu/dû avoir une interjection dans une énonciation. (D. Vincent, p. 72)

Les particules discursives sont traditionnellement décrites par la littérature comme des unités au placement aléatoire, dont la production serait impossible à présupposer. Ces affirmations sont largement à reconsidérer à la lumière des résultats fournis après l'étude des corpus de cinéma choisis ici. Les auteurs ont su prévoir les lieux où les PDi devaient être produites, puisque en se réappropriant le texte, les acteurs en ont reproduit la majorité, voire la totalité pour certaines formes, à l'identique, c'est-à-dire à la même place, et pour le même usage. Et, pour chacune des formes, les acteurs ajoutent beaucoup d'occurrences sur d'autres positions - en plus de celles qui ont été prévues à l'écrit et reprises à l'identique. Ces observations nous conduisent à faire les quatre remarques suivantes :

1. Puisqu'ils écrivent des dialogues dans lesquels on observe les systèmes qui président au fonctionnement de la langue, les auteurs (de ces films là) auraient une vraie prescience des mécanismes de la langue orale.

2. Les PDi ne sont pas produites n'importe comment, sans règles, sans organisation (sinon les auteurs ne pourraient pas les prévoir). Des règles de leur apparition, de leur production peuvent être dégagées.

3. Peu de PDi ne sont pas reprises : ces cas-là sont cependant parmi les plus intéressants. Pourquoi en effet les acteurs les ont-ils « supprimées »? Si chaque occurrence mérite d'être étudiée en détail dans une perspective interactionnelle, nous ne pouvons introduire ce développement dans l'étude présente. Ce point pourra faire l'objet d'un autre travail ciblé.

4. Et a contrario, ce qui n'a pas été écrit, et qui est seulement produit par l'interaction dans le jeu des acteurs, nous parait aussi digne d'intérêt. Essentielles à la formulation, à la structuration, à l'organisation de la parole, les PDi sont largement utilisées (donc utiles) dès qu'un texte doit être reconstruit dans une interaction (même "prévue »), comme c'est le cas lorsque les acteurs se réapproprient un texte. Or, les 
auteurs n'en prévoient jamais assez. Le tableau 1 nous indique en effet qu'un grand nombre de PDi (58 ben, 17 bon, 49 hein, 6 alors) sont produites par les acteurs alors qu'elles n'ont aucune base écrite, qu'elles ne font pas partie du texte qu'ils ont appris. (Là encore, chaque cas serait intéressant à étudier dans un approfondissement des analyses interactionnelles). Les acteurs, en oralisant, construisent un autre texte.

\subsubsection{Les PDi modifient-elles le message ou le style ?}

Les ponctuants étant hautement facultatifs, le découpage est assuré par la prosodie. (D. Vincent, 1993, p. 61 , note 18 )

En évoquant «le caractère hautement facultatif» des éléments qu'elle nomme ponctuants ${ }^{10}$, Diane Vincent « constate finalement que tous les ponctuants pourraient être éliminés sans altérer le message ou le style » (id. p. 62). Nous avons donc examiné les exemples du corpus scénario ne contenant pas de PDi pour les comparer aux énoncés transformés par l'acteur qui se les réapproprie, quand il y a ajouté des PDi : seraient-elles « facultatives » et n'apportent-elles rien dans l'interaction? Pour apporter un premier élément de réponse, voici une réplique issue du scénario de Un Air de Famille écrite pour le personnage Henri joué par JP Bacri. Quand l'acteur l'oralise, il donne une interprétation légèrement différente ; sans changer réellement le texte, il parsème son propos d'éléments supplémentaires et notamment de PDi :

\section{EXEMPLE Un Air de Famille}

Scénario

Henri : À chaque fois, c'est pareil, je la connais la chanson, et que ce soit toi, ou Maman, ou Philippe, c'est toujours la même histoire, je comprends rien, je sais rien, c'est pas la peine de lui expliquer à lui, il est trop con.

Transcription de la production de l'acteur

Henri (Bacri) : Ben + mais à chaque fois c'est pareil je la connais par coeur ta chanson moi + $\boldsymbol{h e i n}$ et que ce soit toi ou Maman ou Philippe + voilà toujours la même histoire euh je comprends rien je sais rien $\boldsymbol{e t} \mathrm{c}$ 'est pas la peine de lui expliquer à lui il est trop con

Ces deux versions illustrent bien à la fois le phonostyle spécifique de l'acteur (dont il ne serait pas pertinent de développer les aspects ici) et une organisation (macro) syntaxique différente des termes, ce qui induit un énoncé plus "coloré », et modifié dans son expressivité. Le message est enrichi des particules discursives (et d'autres termes : moi, par cœur, et...). Les développements fournis par les ajouts, notamment les PDi, apportent à l'interlocuteur des indices nouveaux d'ordre pragmaticodiscursifs, et structurent l'énoncé de manière particulière par rapport au texte qu'il a appris, ce qui hiérarchise les informations, et peut en modifier la réception.

Voici le contexte de la réplique et les tours précédents :

Betty : qu'est-ce que tu veux qu'il te demande tu tu comprends quelque chose toi au marché des ordinateurs toi

Henri : ben oui c'est vrai je suis con moi ben je suis l'imbécile de la famille hein tu as raison je vois vraiment pas pourquoi on me demanderait quelque chose à moi

Betty : est-ce que j'ai dit ça

Henri : ben - mais à chaque fois c'est pareil je la connais par coeur ta chanson moi - hein et que ce soit toi ou Maman ou Philippe - voilà toujours la même histoire euh je comprends rien je sais rien et c'est pas la peine de lui expliquer à lui il est trop con

Betty : mais non mais arrête avec ça c'est pas vrai

Nous laissons de côté une description approfondie des éléments non PDi ajoutés dans cette dernière réplique lors de la production de l'acteur, et nous ne ferons que quelques remarques plus détaillées sur ben et voilà, PDi prononcées par Bacri ici, non prévues dans le scénario. 
Ben, qui est toujours pré-noyau (Teston-Bonnard, 2006), est le signe qu'une nouvelle information va être donnée, et permet à Henri-Bacri de lancer la réplique.
[...] les cas où un locuteur dans son tour de parole marque par ben le début d'une information nouvelle apportée sur un thème déjà présent, et ceux où plusieurs locuteurs accrochent des développements successifs différents sur un même thème en introduisant leur apport par ben. (Traverso \& Bruxelles, p. 52)

Par ce ben anaphorique, l'acteur valide sa précédente réplique qui contenait le message : « tout le monde me traite comme un imbécile ». Il va reprendre l'idée de départ en en donnant une explication, en apportant des détails argumentatifs puisque Betty vient de la contester (est-ce que j'ai dit ça ?)

La valeur de voilà ici, est à la fois conclusive et présentative, ce qui rejoint les analyses de la littérature : selon Léard (1992: 151), voilà n'a pas seulement un rôle conclusif, il «constitue un acte illocutoire », «présente un argument», «ponctue les phases de l'activité » « structure la conversation ».

\begin{abstract}
Voilà marqueur discursif : les emplois absolus en discours gardent des valeurs de bornage et donc d'aspect, qui concernent cette fois le discours dans son ensemble (début, fin). Cela n'exclut pas les valeurs déictiques, voilà pointant le discours passé ou à venir. Ces traits sémantiques ont des conséquences en pragmatique (terme $=$ satisfaction de finir, conformité à l'annonce ou aux prévisions). (Léard, $1992: 151$ )
\end{abstract}

Pour reprendre le message «on m'a toujours traité comme un imbécile », le personnage Henri commence par poser des «preuves» (= je le sais depuis toujours, l'ensemble de la famille a la même attitude d'ailleurs), puis il produit un voilà qui, à la fois, montre qu'il y a une mise au point (valeur conclusive) et que des assertions plus détaillées vont suivre (valeur présentative); «je comprends rien je sais rien et c'est pas la peine de lui expliquer à lui il est trop con».

Lorsque l'acteur agrémente le texte de PDi, il ne s'agit pas seulement d'une «ponctuation » orale pour « faire plus vrai », qui n'apporterait rien d'utile au dialogue : en fait, en se réappropriant le texte, l'acteur accomplit un travail de création qui complète, et accroît véritablement celui des auteurs.

\title{
2.1.3 Les PDi qui ont valeur de tour
}

Certaines particules discursives sont encore plus « essentielles » à la construction de la parole en cours, dans la mesure où elles ont une valeur de tour. Sur le plan interactionnel, elles réalisent alors des actions spécifiques. Sur le plan syntaxique, elles sont des noyaux macro-syntaxiques.Voici deux exemples issus du film Le Goût des Autres, dans lesquels le voilà de J.-P. Bacri et le eh ben tiens de Lanvin sont des unités essentielles à l'interaction.

Le Goût des Autres

(Castella lit le poème qu'il a écrit à l'attention de Clara)

Castella (Il replie la feuille qu'il garde à la main) : Voilà.

Clara : Mmmh...Vous faites des progrès

Deschamps : ouais - en même temps elle me dit pas que c'est fini c'est ça qui me

Moreno : eh ben tiens

Deschamps : non attends quand j'en ai parlé avec Manie elle elle m'a dit qu'une femme [...]

Sur le noyau, que nous définissons comme une unité de communication suffisante et autonome, l'interlocuteur peut s'appuyer et poursuivre par une (ré)action discursive : l'opposition, la contradiction, la confirmation, l'orientation du discours dans une nouvelle direction etc., et invite ainsi à créer à son tour une autre unité communicative.

-Eh ben tiens, qu'on pourrait gloser ici par tu penses! ou bien sûr!, constitue un tour de parole: Deschamps s'appuie sur cet énoncé pour répondre et, en l'occurrence, contredire Moreno. Ici, macrosyntaxiquement eh ben tiens est une unité discursive autonome et il s'agit bien d'un «noyau » (voir infra 
et la note 11); sur le plan de l'interaction, il crée une action qui provoque le tour suivant, la réponse contradictoire de Deschamps (non atttends...).

-En produisant voilà, Castella indique que son poème est terminé, qu'il a fini de lire. En contexte, cependant, nous savons que ce voilà ne marque pas seulement la fin de sa lecture. À travers ce poème, il a déclaré ses sentiments à Clara. Voilà, ici, est aussi chargé de l'aveu qu'il vient de faire : il est ainsi porteur d'une attente et d'une ouverture. Fournissant des indices instructionnels qui sont fondamentaux pour la compréhension de la situation, il a aussi valeur de tour : Clara prend alors la parole pour réagir aux déclarations sentimentales de Castella. Elle refuse de répondre directement à sa question sous-jacente (= m'aimez-vous ?) en poursuivant l'échange comme une situation professionnelle (vous faites des progrès). Voilà a créé une action préparatrice de tour, en contribuant à solliciter la réponse de Clara.

\subsubsection{Les PDi sont-elles interchangeables?}

Traiter les ponctuants comme une variable implique nécessairement que les variantes sont équivalentes, selon un critère ou un autre. Mais sont-elles substituables les une aux autres?

Devons-nous être étonnés de constater que la substitution n'est pas toujours possible (D. Vincent, 1993 : 77).

Si les PDi étaient des éléments interchangeables, qu'elles n'étaient que des marques sans valeurs pragmatiques spécifiques, n'importe quelle PDi devrait pouvoir s'insérer dans n'importe quel énoncé. Examinons deux versions différentes de la même réplique dans Le Goût des Autres : la version écrite ne contient pas de PDi; dans la version oralisée, Gérard Lanvin qui interprète le personnage de Moreno, ajoute la PDi bon:

\section{Le Goût des Autres}

Scénario

Moreno : je fais quoi comme tête? Je vais pas hurler de rire parce que je te vois vendre cette merde...

Manie : (dos caméra) « cette merde »... ?)

Moreno : (après un temps)...Je vais pas tarder à y aller, moi...(Il range ses cigarettes dans la poche de sa chemise)

\section{Transcription}

Moreno : je fais quoi comme tête je vais pas hurler de rire parce que je te vois vendre cette merde +

Manie : cette merde +

Moreno : bon je vais pas tarder à y aller moi

En oralisant la réplique écrite dans le scénario, qui exprime une colère et une désapprobation telles que le personnage préfère clore l'entretien et partir, l'acteur introduit son énoncé par la PDi qui semble la plus pertinente dans la situation: bon. Les valeurs pragmatico-discursives de cette PDi correspondent à un point de transition de l'interaction puisque bon, qui est résomptif et incorpore les actions des tours précédents (Brémond, C., 2002), -il est alors paraphrasable par « puisque c'est comme ça » «j'ai compris, OK!» (ici bon = c'est bon)-, ouvre ici un nouvel épisode de l'échange. Le rôle spécifique de cette particule-là, au niveau organisationnel et structurant, est particulièrement clair. Pour faire la jonction entre les deux séquences interactionnelles, -l'échange conflictuel avec Manie, et la décision de stopper l'échange en partant-, quelle autre particule aurait pu convenir à la situation pour indiquer à la fois la prise en compte du conflit et l'ouverture vers une autre étape, le départ ? Ben, alors, ou enfin porteraient des valeurs différentes et construiraient l'échange différemment. L'étude des versions différentes issues de l'écriture du texte (scénario) et de la production des acteurs (transcription), nous permettent de confirmer que les PDi ont des valeurs pragmatiques et interactionnelles tout à fait spécifiques par forme, et qu'elles ne sont ni écrites, ni produites au hasard. 


\subsection{Statut syntaxique des PDi}

Les usages interactionnels et discursifs indiquent donc que les PDi font partie d'un domaine organisé, c'est-à-dire qu'elles sont soumises à des régularités et à des contraintes systémiques-, et qu'elles sont noyaux quand elles ont valeur de tour. Dès lors, il n'est plus possible de refuser une analyse syntaxique, alors même que les grammaires ne les ont pas (ou si mal) intégrées dans les descriptions traditionnelles.

Notre travail se place dans la perspective de la syntaxe interprétée au sens large, qui se définit par extension comme l'ordonnance des éléments du discours. Le modèle théorique de la macro-syntaxe aixoise (cf supra : \&1.3. et 2.1.3.) par lequel nous exploitons des unités de référence différentes (versus phrase et proposition), le noyau, le préfixe, les suffixe et postfixe, et l'unité que nous ajoutons au modèle, l'infixe $^{11}$. La description macro-syntaxique ne doit cependant pas bloquer l'observation des relations contraintes des PDi avec la micro-grammaire des catégories.

\subsubsection{Critères syntaxico-discursifs définitoires délimitant une classe PDi}

Entrent dans la classe PDi les petits mots et constructions verbales du discours bon, ben, quoi, alors, voilà, enfin, là, tu sais, tu vois, je veux dire etc. qui ne sont pas utilisés avec le sens prioritairement ou exclusivement donné par les dictionnaires et les approches traditionnelles de la grammaire, qui grammaticalement ne sont pas rattachés aux autres termes de l'énoncé dans une relation de dépendance (non régis), et sont affectés de restrictions distributionnelles et topologiques d'insertion et de contraintes d'ordre macro-syntaxique (syntaxe externe : régularités de configurations); enfin, ils sont soumis à des contraintes au niveau des combinatoires en syntaxe interne (Teston-Bonnard, 2006). Ils sont porteurs de valeurs instructionnelles construites dans l'interaction, et non référentielles.

\subsubsection{Distribution et topologie : propriétés catégorielles.}

Nous observons que certaines formes occupent plus systématiquement certaines places macrosyntaxiques que d'autres : si les tableaux 2 et 2 ' montrent que bon, alors, et ben sont plutôt des prénoyaux, que voilà est plus souvent noyau, et que enfin peut occuper de manière à peu près équivalente les places de pré et post-noyaux, il est particulièrement intéressant d'observer que ces régularités se retrouvent dans les deux corpus (dialogue des scénaristes et oral des acteurs). Les fréquences sont beaucoup plus importantes pour le second corpus, mais dans les mêmes proportions.

Par ailleurs, si certaines formes occupent très rarement (ou pas du tout) certaines positions (ben postnoyau ou voilà intra-noyau), là encore les deux corpus révèlent les mêmes tendances.

L'étude spécifique des intra-noyaux montre des blocages systémiques et récurrents d'insertions des PDi par rapport aux catégories grammaticales : par exemple, il n'est pas observé d'insertions entre clitique et verbe, entre déterminant et nom, entre adjectif antéposé et le nom, etc. (Teston-Bonnard, 2006), et des insertions restreintes ou inexistantes là où des adverbes comme quand même ou évidemment s'insèrent facilement. Ces propriétés distributionnelles, relevées dans nos corpus de cinéma, sont similaires à celles qui ont été repérées dans les autres corpus de données authentiques (Teston-Bonnard, 2006). Enfin, les restrictions en termes de syntaxe interne (pas d'expansion du syntagme dont la PDi est la tête, ou expansion limitée) sont systématiques pour toutes les formes. Elles sont également observables dans l'ensemble des corpus étudiés.

Les contraintes régissant ce type d'organisation sont bien d'ordre syntaxique et pas seulement d'ordre pragmatique. Qu'il s'agisse de la formulation de PDi prévue par les auteurs ou de celle des acteurs, l'examen des corpus de cinéma, nous permet de retrouver chaque fois la même organisation syntaxique.

\subsubsection{Propriétés fonctionnelles (macro)syntaxiques des PDi}

Dans les autres corpus de données authentiques comme dans les corpus de cinéma, le statut catégoriel en termes de distribution et de topologie (pré-noyau, post-noyau, intra-noyau, noyau), attribué aux PDi étudiées, correspond à différentes propriétés fonctionnelles de type syntaxique (Teston-Bonnard, 2006) : 
$1^{\circ}$ ) Les fonctions des pré-noyaux

-embrayeurs elles « lancent» une unité ;

L1 oui mais ça c'est ce que tu fais en cours mais toi qu'est-ce que tu penses de ça justement

L2 ben moi en cours je me plie à aux règles donc euh j'utilise ça parce que je tiens à avoir des notes raisonnables (Corpaix. 11FRAGRA)

-structurants, elles marquent une unité à l'intérieur d'une autre unité : une parenthèse, un segment de discours rapporté ; elles sélectionnent particulièrement un noyau pour l'interlocuteur.

+ pareil pour euh remuer la salade + il y en a qui disent touiller il y en a qui disent mélanger bon ça c'est vraiment les plus compréhensibles mais il doit y avoir des mots qu'on (ne, Ø) comprend pas dans certaines régions je les ai pas tous en tête mais + ça doit être très différent d'une région à l'autre hein (Corpaix. 11FRAG)

+ et quand sa mère est revenue tout était fait + et là c'est vraiment une situation ridicule + où j'ai é- essayé de me remettre en question + et où forte de mes valeurs et de mes concepts je me suis dit ben oui c'est un échec + (CRFP. PRI-AMI-2)

-recatégorisateurs (d'elles-mêmes), elles constituent contextuellement un noyau, alors qu'elles sont prototypiquement pré-noyaux.

- dis-moi la vérité tu as eu une mauvaise note

- ben

- oh encore mais tu vas te mettre à travailler quand (Corpus personnel)

$2^{\circ}$ ) Fonction des intra-noyaux

-décondensateurs (Morel, MA.,1995), elles re-segmentent une unité noyau en mettant différemment en exergue les séquences de ce noyau.

+ ils me faisaient quoi la remarque mais gentiment (Corpaix. Portugas)

$3^{\circ}$ ) Les fonctions des post-noyaux

-désignateurs, et recatégorisateurs (d'une unité dans laquelle elles se trouvent), elles permettent de donner un autre statut à une unité habituellement interprétée différemment : par exemple, à la fin d'une unité pré-noyau, elles catégorisent contextuellement celle-ci comme un noyau; elles indiquent le statut de l'unité précédente.

[mais euh quand on voit euh David Douillet par exemple qui est l'exemple suprême du judoka et qui a trente ans et qui s'apprête à faire les euh + Jeux Olympiques de Sydney quoi] + donc euh + mais enfin bon il y a il y a de plus en plus de problèmes enfin moi j'ai vu un article * sur le journal là il y a pas longtemps + où euh ils parlent de dopage dans le Judo qui s'est qui édeviendrait une une pratique de plus en plus courante + donc après on sait pas trop euh sur quoi euh on se base pour euh pour dire lui il est + il est fort quoi (CRFP. PRI-BOR 1)

$4^{\circ}$ ) Fonction des PDi qui se situent entre deux unités (pré-noyau ou post-noyau)

-connecteurs, elles établissent un lien discursif entre deux unités.

la deuxième langue qui est normalement le plus parlé(e) ben c'est le l'anglais (Corpaix. 12FRAN)

$5^{\circ}$ ) Fonction des PDi sans position contrainte

-noyaux à part entière, elles constituent des unités autonomes discursivement par effet de greffe (Deulofeu, 1989, 1999) ou parce qu'elles ont valeur de tour.

Deschamps : et euh - non

Manie : ben quoi vas-y

Deschamps : non

Manie : bon

Deschamps : non mais ça t'embêterait qu'on se revoit un de ces jours /là, $\mathrm{O} /$ - comme ça pour parler hein (Le Goût des Autres) 


\subsubsection{Typologie fondée sur la combinaison des analyses micro et macro-syntaxiques}

Nous donnons les résultats par catégories et fonctions des PDi les plus souvent repérées dans Le Goût des Autres, en comparant le texte du scénario et la réappropriation du texte quand les acteurs l'oralisent.

Tableaux Comparatifs Scénario/ Transcription du film Le Goût des Autres

Scénario Tableau 2

\begin{tabular}{|c|c|c|c|c|c|c|c|c|}
\hline Catégorie & Fonction & hein & ben & alors & Voilà & enfin & bon & Tiens \\
\hline PRE-NOYAU & $\begin{array}{l}\text { Embrayeur de } \\
\text { Noyau }\end{array}$ & & 40 & 15 & 2 & 5 & 17 & 1 \\
\hline $\begin{array}{l}\text { POST-NOYAU } 1 \\
\text { Finale simple }\end{array}$ & $\begin{array}{l}\text { Désignation } \\
\text { de noyau }\end{array}$ & 27 & & 5 & 2 & 1 & & \\
\hline $\begin{array}{l}\text { PRE-NOYAU } \\
\text { sans noyau réalisé }\end{array}$ & $\begin{array}{l}\text { Recatégorisateur } \\
\text { Parfois Noyau }\end{array}$ & & 1 & & & 1 & & \\
\hline \multirow{3}{*}{$\begin{array}{l}\text { PRE-NOYAU } \\
\text { STRUCTURANTS } \\
\text { Marque des } \\
\text { dispositifs } \\
\text { macro }\end{array}$} & M. d'incise & & & & & & 2 & \\
\hline & $\begin{array}{l}\text { M.de } \\
\text { D.Rapporté }\end{array}$ & 1 & 1 & & & & & \\
\hline & $\begin{array}{l}\text { M. de } \\
\text { saillance }\end{array}$ & 1 & 6 & 4 & & & & \\
\hline \multirow{2}{*}{$\begin{array}{l}\text { NOYAU } \\
\text { unité de } \\
\text { communication }\end{array}$} & greffe & & & & & 1 & 1 & \\
\hline & noyau & 7 & 1 & 1 & 6 & 3 & 4 & 1 \\
\hline INTRA-NOYAU & $\begin{array}{l}\text { Décondensation } \\
\text { De noyau }\end{array}$ & 3 & & & & 1 & & \\
\hline $\begin{array}{l}\text { PRE-NOYAU ou } \\
\text { POST-NOYAU } 2 \\
(=\text { suffixe) }\end{array}$ & Connecteur & 5 & 2 & 2 & 5 & 6 & 2 & 1 \\
\hline
\end{tabular}

Transcription du film Tableau 2'

\begin{tabular}{|c|c|c|c|c|c|c|c|c|}
\hline & & hein & ben & alors & Voilà & enfin & bon & Tiens \\
\hline PRE-NOYAU & $\begin{array}{l}\text { Embrayeur de } \\
\text { Noyau }\end{array}$ & & 71 & 20 & 4 & 10 & 22 & 1 \\
\hline $\begin{array}{l}\text { POST-NOYAU } 1 \\
\text { Finale simple }\end{array}$ & $\begin{array}{l}\text { Désignation } \\
\text { de noyau }\end{array}$ & 56 & & 6 & 4 & 2 & & \\
\hline $\begin{array}{l}\text { PRE-NOYAU } \\
\text { sans noyau réalisé }\end{array}$ & $\begin{array}{l}\text { Recatégorisateu } \\
\text { Parfois noyau }\end{array}$ & & & & & 1 & & \\
\hline \multirow{3}{*}{$\begin{array}{l}\text { PRE-NOYAU } \\
\text { STRUCTURANT } \\
\text { Marque des } \\
\text { dispositifs } \\
\text { macro }\end{array}$} & M. d'incise & & & & & & 2 & \\
\hline & $\begin{array}{l}\text { M.de } \\
\text { D. Rapporté }\end{array}$ & 1 & 1 & & & & & \\
\hline & $\begin{array}{l}\text { M. de } \\
\text { Saillance }\end{array}$ & 2 & 7 & 5 & & & & \\
\hline
\end{tabular}




\begin{tabular}{|l|l|l|l|l|l|l|l|l|}
\hline $\begin{array}{l}\text { NOYAU } \\
\text { unité de } \\
\text { communication }\end{array}$ & Greffe & & & & & & 1 & \\
\cline { 2 - 8 } & Noyau & 6 & & 3 & $\mathbf{6}$ & 1 & 3 & 1 \\
\hline INTRA-NOYAU & $\begin{array}{l}\text { Décondensation } \\
\text { De noyau }\end{array}$ & 8 & & 1 & & & 1 & 1 \\
\hline $\begin{array}{l}\text { PRE-NOYAU ou } \\
\text { POST-NOYAU 2 } \\
(=\text { suffixe })\end{array}$ & Connecteur & 19 & 7 & 4 & 1 & 7 & 3 & \\
\hline
\end{tabular}

\section{Conclusion}

L'examen de la langue cinématographique dans ses deux aspects, la version scénarisée (écrite par les auteurs) et l'oralisation (jeu des acteurs), et l'étude plus approfondie de certaines unités interprétées comme étant propres à l'oral - les PDi - montrent que la langue s'élabore ici dans une coexistence fusionnelle des deux pratiques du langage, telles qu'elles ont été étudiées dans leurs usages attestés. Cette coexistence, ou plus précisément cette coalescence (Teston-Bonnard, 2004), organise la langue cinématographique en un continuum de relations réciproques entre les interactions prévues et oralisées. Ici, les auteurs imaginent et représentent un langage qui doit être reçu comme un discours fabriqué au fur et à mesure ; le genre (comédies-chroniques quotidiennes) dans lequel se situent les scénarios étudiés ici ne sont que très peu composés de monologue et de récit : il s'agit plutôt de conversations à bâton rompu au cours desquelles les personnages sont censés s'interrompre, s'interpeller, s'interroger, se répondre vivement.

On a pu constater, à travers l'analyse plus spécifique des PDi, que les auteurs, puis les acteurs ont ici une bonne intuition des caractéristiques connues du langage en interaction décrites par de nombreux spécialistes, puisque les repérages effectués sur corpus de cinéma fournissent des résultats proches de ceux réalisés auparavant sur les autres corpus de données attestées de l'oral. Or, cette proximité ne les éloigne pas complètement de la norme grammaticale. Nous confirmons que la norme grammaticale de la langue écrite marque ici subtilement le scénario et sa production; ainsi, l'effort des auteurs vers une représentation juste de l'oral provient à la fois de leur perception subjective de la langue parlée -parfois proche de la réalité, parfois peut être issue d'une démarche stéréotypée-, et aussi des contraintes conscientes et inconscientes imposées par leurs savoirs culturels.

Enfin, -et cette observation est sans doute la plus marquante- on doit en conclure que les interactions entre acteurs au cinéma sont aussi fécondes que les interactions entre locuteurs dans des situations quotidiennes, ce qui indique que l'échange entre acteurs, à partir d'un texte écrit et appris, reste un véritable langage en interaction et constitue une situation de parole réelle ( $v s$ fictive) au même titre que celles qui sont étudiées habituellement au travers des corpus de données authentiques.

Il nous paraît intéressant de poursuivre ce type d'étude sur d'autres corpus de cinéma pour enrichir les résultats obtenus dans cette contribution; en outre, l'étude du statut des PDi peut être encore développée parce que ces unités constituent, pour les auteurs et les acteurs -qui achèvent le travail de création des scénaristes-, à la fois une ressource utile à la «fabrication» d'une langue de genre, et, pour les chercheurs, un indice pointant les différentes situations d'interactions du français parlé.

Enfin, l'étude de la perception du spectateur qui reçoit les dialogues comme naturels ou non permettrait de poursuivre le travail de recherche vers une meilleure compréhension du fonctionnement de la langue dans tous ses aspects.

\section{Références Bibliographiques}

Béguelin, M.-J., (dir.) (2000). De la phrase aux énoncés: grammaire scolaire et descriptions linguistiques. Bruxelles : De Boeck. 
Blanche-Benveniste C., (1990). Études Grammaticales, Le Français Parlé. Paris : CNRS.

Blanche-Benveniste C., (1997). Approches de la Langue Parlée en français. Paris, Gap : Ophrys.

Brémond, C. (2002). Les petites marques du discours. Le cas du marqueur méta-discursif «bon» en français (06/12/02) Thèse Direction Robert Vion, Université de Provence.

Bruxelles, S., Traverso, V., (2001). Ben dans deux situations polylogales. Apport de la description d'un "petit mot" du

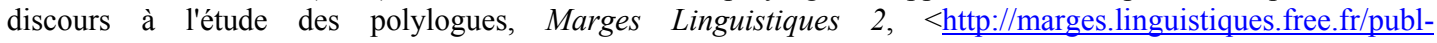
act/publactl.htm>.

Bruxelles S., Traverso V., (2005, à paraître). Usages de la particule voilà dans une réunion de travail : analyse multimodale. In Drescher M. \& Job B. (ed), Les marqueurs discursifs dans les langues romanes : approches théoriques et méthodologiques. Francfort : Peter Lang.

Chanet, C., (2004). Fréquence des marqueurs discursifs en français parlé : quelques problèmes de méthodologie. RSFP $18: 83-106$

Chion, M., (2008). Le complexe de Cyrano. La langue parlée dans les films français. Paris : Editions Cahiers du cinéma. $190 \mathrm{p}$.

Deulofeu, J. (1989). Les couplages de constructions verbales en français parlé : effet de cohésion discursive ou syntaxe de l'énoncé. Recherches sur le français parlé 9, 111-141.

Deulofeu, J., (1999). Recherches sur les formes de la prédication dans les énoncés assertifs en français contemporain (le cas des énoncés introduits par le morphème que). Thèse d'Etat, Université Paris III.

Ducrot, O., (1980). Les Mots du Discours. Paris : Editions de Minuit.

Goosse, A., (1993), Le Bon Usage Grevisse. Paris, Louvain-la-Neuve : Duculot (13 $3^{\text {ème }}$ édition).

Léard, J.-M., (1992). Les Gallicismes. Étude syntaxique et sémantique. Paris-Louvain-La-Neuve : Duculot.

Mondada, L., (2004). Temporalité, séquentialité et multimodalité au fondement de l'organisation de l'interaction : Le pointage comme pratique de prise du tour. Cahiers de Linguistique Française, 26, 169-192.

Mondada, L., (2001). Pour une linguistique interactionnelle, Marges Linguistiques, 1, mai, 142-162 (archive accessible sur Internet: http://www.revue-texto.net/Archives/Archives.htm ) (republié dans M. Santacroce, éd., 2001, Faits de langue, faits de discours, 2, Paris: L 'Harmattan, 95-136) (trad. esp. Por una lingüística interaccional, Discurso y Sociedad, 3/3, 2001, 61-90).

Mondada, L., (1995). Planification syntaxique des énoncés et séquentialité de la conversation, SCOLIA (Actes du Colloque BENEFRI-Strasbourg, Problèmes de sémantique et de relations entre micro- et macro-syntaxe, Neuchâtel, 19-21 mai 1994), 5, 319-342.

Riegel, M., Pellat, J.C., Rioul, R., (1994). Grammaire méthodique du français. Paris : PUF.

Ryngaert, J.-P., (1993). Lire le théâtre contemporain. Paris : Dunod.

Teston-Bonnard, S., (2004). L'écriture au cinéma comme représentation du langage parlé. L'Écriture dans tous ses états, Approches en sciences cognitives, 251-269. Aix-en-Provence (mai 2003). Prix de la meilleure conférence.

Teston-Bonnard, S., (2006). Propriétés topologiques et distributionnelles des constituants non régis. Application à une description syntaxique des particules discursives. Doctorat de Linguistique, Université de Provence, UFR Lettres, Arts, Communication et Sciences du Langage (L.A.C.S.). Soutenu le 08 décembre 2006 à l'Université de Provence (Aix en Provence) sous la direction de Henri-José Deulofeu.

Teston-Bonnard, S., (2007). « Ruptures » dans le dialogue de cinéma. In Broth, M., Forsgren, M., Norén, C. \& SulletNylander, F. (eds). Le français parlé des médias.

Traverso V., 1999, L'Analyse des conversations. Paris : Nathan Coll. 128.

Vincent, D., (1993). Les ponctuants de la langue et autres mots du discours. Québec : Nuit Blanche

\footnotetext{
${ }^{1}$ Puisqu'il existe aussi des dialogues en vers pour des adaptations d'œuvres théâtrales.
} 
${ }^{2}$ Même dans les scénarios d'Audiard, dont les dialogues qui paraissent si naturels aux spectateurs, sont en réalité tellement difficiles à se réapproprier ! -d'après les acteurs qui le jouent-.

${ }^{3}$ Ce que Chion nomme le style neutre est un style standard (voir le chapitre 1 de son ouvrage, cité en référence)

${ }^{4}$ Une plate forme de données telle que CLAPI, qui est constitué de corpus d'enregistrements oraux réalisés en situation avec le moins de contraintes possibles imposées par le chercheur qui enregistre, en audio et en vidéo, et traite les données avec des outils destinés à l'observation des interactions; CRFP et Corpaix, $(2$ corpus $=1$ million et demi de mots), entretiens et conversations d'ordre privé, public et professionnel, enregistrés et transcrits par les chercheurs aixois du GARS/DELIC sur toute la France.

${ }^{5}$ Pour Un Air de Famille, un manuscrit non publié nous a été remis par les agents de Jaoui-Bacri, pour Le Goût des Autres, nous avons exploité le texte publié par l'Avant Scène Cinéma.

${ }^{6}$ Groupement Aixois de Recherche en Syntaxe, dirigé par C. Blanche Benveniste jusqu'en 1999.

${ }^{7}$ Il y a eu les essais dramaturgiques ou cinématographiques réalisés dans ce sens : certains films, certaines entreprises théâtrales «d'auteur», que nous ne citerons pas ici, sont davantage des démonstrations expérimentales que des œuvres artistiques...

${ }^{8}$ Les dictionnaires le classent comme interjection ou comme adverbe.

${ }^{9}$ Notamment Chanet (2004) montre que bien n'a pas les mêmes fréquences d'apparition et ne se trouve pas dans les mêmes registres d'oraux que ben.

${ }^{10}$ Il s'agit pour D. Vincent d'un sous-type de particule discursive, dans lequel elle intègre des formes comme je veux dire, là, tu sais, etc.

${ }^{11}$ Nous choisissons, à l'instar d'autres auteurs, de nommer ces unités plutôt « pré-noyau », « post-noyau » et « intranoyau ». 